\title{
Prediction of Glucose Intolerance in Early Postpartum in Women with Gestational Diabetes Mellitus Based on the 2013 WHO Criteria
}

Katrien Benhalima ${ }^{1, *} \mathbb{0}$, Paul Van Crombrugge ${ }^{2}$, Carolien Moyson ${ }^{1}$, Johan Verhaeghe ${ }^{3}$, Sofie Vandeginste ${ }^{4}$, Hilde Verlaenen ${ }^{4}$, Chris Vercammen ${ }^{5}$, Toon Maes ${ }^{5}$, Els Dufraimont ${ }^{6}$, Christophe De Block ${ }^{7}$, Yves Jacquemyn ${ }^{8}$, Farah Mekahli ${ }^{9}$, Katrien De Clippel ${ }^{10}$, Annick Van Den Bruel ${ }^{11}$, Anne Loccufier ${ }^{12}$, Annouschka Laenen ${ }^{13}$, Caro Minschart ${ }^{1}$, Roland Devlieger ${ }^{3}$ (I) and Chantal Mathieu ${ }^{1}$

1 Department of Endocrinology, University Hospital Gasthuisberg, KU Leuven, Herestraat 49, 3000 Leuven, Belgium; carolien.moyson@uzleuven.be (C.M.); caro.minschart@kuleuven.be (C.M.); chantal.mathieu@uzleuven.be (C.M.)

2 Department of Endocrinology, OLV Ziekenhuis Aalst-Asse-Ninove, Moorselbaan 164, 9300 Aalst, Belgium; Paul.Van.Crombrugge@olvz-aalst.be

3 Department of Obstetrics \& Gynecology, University Hospital Gasthuisberg, KU Leuven, Herestraat 49, 3000 Leuven, Belgium; johan.verhaeghe@uzleuven.be (J.V.); roland.devlieger@uzleuven.be (R.D.)

4 Department of Obstetrics \& Gynecology, OLV Ziekenhuis Aalst-Asse-Ninove, Moorselbaan 164, 9300 Aalst, Belgium; Sofie.Vandeginste@olvz-aalst.be (S.V.); Hilde.Verlaenen@olvz-aalst.be (H.V.)

5 Department of Endocrinology, Imelda Ziekenhuis, Imeldalaan 9, 2820 Bonheiden, Belgium; Chris.Vercammen@imelda.be (C.V.); Toon.Maes@imelda.be (T.M.)

6 Department of Obstetrics \& Gynecology, Imelda Ziekenhuis, Imeldalaan 9, 2820 Bonheiden, Belgium; Els.Dufraimont@imelda.be

7 Department of Endocrinology-Diabetology-Metabolism, Antwerp University Hospital, Wilrijkstraat 10, 2560 Edegem, Belgium; Christophe.DeBlock@uza.be

8 Department of Obstetrics \& Gynecology, Antwerp University Hospital, Wilrijkstraat 10, 2560 Edegem, Belgium; Yves.Jacquemyn@uza.be

9 Department of Endocrinology, Kliniek St-Jan Brussel, Kruidtuinlaan 32, 1000 Brussel, Belgium; fmekahli@clstjean.be

10 Department of Obstetrics \& Gynecology, Kliniek St-Jan Brussel, Kruidtuinlaan 32, 1000 Brussel, Belgium; kdeclippel@gmail.com

11 Department of Endocrinology, AZ St Jan Brugge, Ruddershove 10, 8000 Brugge, Belgium; Annick.VandenBruel@azsintjan.be

12 Department of Obstetrics \& Gynecology, AZ St Jan Brugge, Ruddershove 10, 8000 Brugge, Belgium; anne.loccufier@azsintjan.be

13 Center of Biostatics and Statistical bioinformatics, KU Leuven, Kapucijnenvoer 35 bloc d-box 7001, 3000 Leuven, Belgium; annouschka.laenen@kuleuven.be

* Correspondence: katrien.benhalima@uzleuven.be; Tel.: +32-16-340614

Received: 7 February 2019; Accepted: 15 March 2019; Published: 19 March 2019

Abstract: Predictors for glucose intolerance postpartum were evaluated in women with gestational diabetes mellitus (GDM) based on the 2013 World Health Organization (WHO) criteria. 1841 women were tested for GDM in a prospective cohort study. A postpartum $75 \mathrm{~g}$ oral glucose tolerance test (OGTT) was performed in women with GDM at $14 \pm 4.1$ weeks. Of all 231 mothers with GDM, 83.1\% (192) had a postpartum OGTT of which $18.2 \%$ (35) had glucose intolerance. Women with glucose intolerance were more often of Asian origin [15.1\% vs. 3.7\%, OR 4.64 (1.26-17.12)], had more often a recurrent history of GDM [41.7\% vs. $26.7 \%$, OR 3.68 (1.37-9.87)], higher fasting glycaemia (FPG) [5.1 (4.5-5.3) vs. 4.6 (4.3-5.1) mmol/L, OR 1.05 (1.01-1.09)], higher HbA1c [33 (31-36) vs. 32 (30-33) $\mathrm{mmol} / \mathrm{mol}$, OR 4.89 (1.61-14.82)], and higher triglycerides [2.2 (1.9-2.8) vs. 2.0 (1.6-2.5) $\mathrm{mmol} / \mathrm{L}$, OR 1.00 (1.00-1.01)]. Sensitivity of glucose challenge test (GCT) $\geq 7.2 \mathrm{mmol} / 1$ for glucose intolerance 
postpartum was $80 \%(63.1 \%-91.6 \%)$. The area under the curve to predict glucose intolerance was 0.76 (0.65-0.87) for FPG, 0.54 (0.43-0.65) for HbA1c and 0.75 (0.64-0.86) for both combined. In conclusion, nearly one-fifth of women with GDM have glucose intolerance postpartum. A GCT $\geq 7.2 \mathrm{mmol} / \mathrm{L}$ identifies a high risk population for glucose intolerance postpartum.

Keywords: glucose intolerance; postpartum; gestational diabetes mellitus; 2013 WHO criteria; risk factors; prediction

\section{Introduction}

Gestational diabetes mellitus (GDM) is defined as diabetes diagnosed in the second or third trimester of pregnancy provided that overt diabetes early in pregnancy has been excluded [1]. Treatment of GDM between 24-28 weeks of pregnancy can reduce adverse pregnancy outcomes, especially large-for-gestational age infants and preeclampsia [2,3]. Women with GDM have a seven-fold increased risk of developing type 2 diabetes (T2DM) later in life compared to normal glucose tolerant (NGT) women during pregnancy [4,5]. Women with persistent glucose intolerance [impaired fasting glucose (IFG) and/or impaired glucose tolerance (IGT)] in early postpartum are a particularly high risk group, with about 50\% developing T2DM within 5 years after the delivery [6]. Long-term follow-up is often challenging due to the low attendance rates at screening tests postpartum [7].

The 'International Association of Diabetes and Pregnancy Study Groups' (IADPSG) and the World Health Organization (WHO) recommend a universal one-step approach with the $75 \mathrm{~g}$ oral glucose tolerance test (OGTT) for screening of GDM with the use of more stringent diagnostic criteria for GDM [8,9]. The IADPSG/2013 WHO criteria are the first diagnostic criteria based on adverse pregnancy outcomes $[8,10]$. However, data on the risk to develop glucose intolerance postpartum in women with GDM diagnosed by the one-step approach and 2013 WHO criteria, are limited. The use of the 2013 WHO criteria for GDM results in a greater proportion of women diagnosed with mild forms of GDM. This might lead to a lower proportion at risk for postpartum glucose intolerance compared to women diagnosed with GDM by a two-step screening strategy [11,12]. In addition, there are currently few data on the clinical and biochemical risk factors which best predict persistent glucose intolerance in early postpartum in women with GDM diagnosed by a universal one-step screening strategy and the 2013 WHO criteria. More evidence is therefore needed on the risk and predictors for glucose intolerance in early postpartum in women with GDM based on the 2013 WHO criteria. Our aim was to evaluate the prevalence and predictors for glucose intolerance in early postpartum in women with GDM from a large prospective cohort study.

\section{Subjects and Methods}

The study was registered in ClinicalTrials.gov (NCT02036619). The study protocol was approved by the Institutional Review Boards of all participating centers (B322201420693). Participants provided informed consent before inclusion in the study.

\subsection{Study Design}

The Belgian Diabetes in Pregnancy study (BEDIP-N) was a multi-centric prospective cohort study that has previously been described in detail [13-15]. Women between 18-45 years with singleton pregnancies, and without history of diabetes or bariatric surgery, were recruited between 6-14 weeks of pregnancy [13]. Participants without prediabetes or diabetes in early pregnancy [defined by the American Diabetes Association (ADA) criteria)], received both a non-fasting 50 g glucose challenge test (GCT) and a $75 \mathrm{~g}$ 2-h oral glucose challenge test (OGTT) between 24-28 weeks of pregnancy $[1,13]$. All participants received the OGTT irrespective of the result of the GCT. The diagnosis of GDM was based on the 2013 WHO criteria. We have recently shown that the threshold of the GCT needs to be 
reduced to at least $7.2 \mathrm{mmol} / \mathrm{L}$, to achieve sensitivity $\geq 70 \%$ to screen for GDM based on the 2013 WHO criteria [14]. The ADA recommended glycemic targets were used for the treatment of GDM [1]. If targets were not achieved with lifestyle measures, insulin therapy was added. Women with GDM were invited for an extra visit 6-16 weeks postpartum to undergo a $75 \mathrm{~g}$ OGTT. The ADA criteria were used to define T2DM and glucose intolerance (IFG and/or IGT) [1,13].

\subsection{Study Assessments}

In early pregnancy, baseline characteristics and the obstetrical history were collected [13]. In early pregnancy and at 24-28 weeks of pregnancy, anthropometric measurements were obtained, a clinical examination was performed [weight, body mass index (BMI) and blood pressure] and several self-administered questionnaires were completed [13]. Overweight was defined as a body mass index (BMI) $\geq 25 \mathrm{Kg} / \mathrm{m}^{2}$ and obesity as a BMI $\geq 30 \mathrm{Kg} / \mathrm{m}^{2}$. Excessive weight gain was defined according to the 2009 Institute of Medicine (IOM) guidelines [16]. Early weight gain was calculated as the difference in weight between first prenatal visit and the time of the OGTT, total weight gain was calculated as the difference in weight between first prenatal visit and the delivery. We used a self-designed questionnaire to evaluate breastfeeding [13]. Women were categorized as: almost exclusive breastfeeding ( $\leq 45 \mathrm{~mL}$ formula feeding/day), half breastfeeding and half formula feeding, and almost exclusive formula feeding ( $\geq 150 \mathrm{~mL}$ formula feeding/day).

At first visit between 6-14 weeks of pregnancy, fasting plasma glucose (FPG), fasting insulin, fasting lipid profile (total cholesterol, HDL- and LDL-cholesterol, triglycerides) and HbA1c were measured. The homeostasis model assessment of insulin resistance (HOMA-IR) and $\beta$-cell function (HOMA-B), were measured in early pregnancy, as previously described [17]. At the time of the $75 \mathrm{~g}$ OGTT during pregnancy and postpartum, a fasting lipid profile and $\mathrm{HbA1c}$ were measured. Glucose and insulin were measured fasting, at $30 \mathrm{~min}, 60 \mathrm{~min}$, and $120 \mathrm{~min}$. For the OGTT, participants had to be fasting for at least $10 \mathrm{~h}$. Increase in triglycerides was defined as the difference between the fasting triglycerides measured in early pregnancy and at the time of the OGTT in pregnancy [13].

Different indices of insulin sensitivity [the Matsuda index, a well-established measure of whole-body insulin sensitivity and the homeostasis model assessment of insulin resistance (HOMA-IR), a measure of largely hepatic insulin resistance] and $\beta$-cell function [HOMA-B, the insulinogenic index divided by HOMA-IR, Stumvoll index and the insulin secretion-sensitivity index-2 (ISSI-2), an OGTT-derived measure that is analogous to the disposition index obtained from the frequently sampled intravenous glucose tolerance test], were measured, as previously described [13,17-22]. The Matsuda and Stumvoll indices were multiplied to calculate the oral disposition index, which assesses $\beta$-cell compensation for insulin resistance [23].

The analyses of the FPG at 6-14 weeks and the glucose measurements of the OGTT were performed locally at each center. The analyzes of the GCT's, insulin, lipids and HbA1c levels were performed centrally at the lab of UZ Leuven (Leuven, Belgium) and these results were not communicated to participants and health care providers during the study. Plasma glucose was measured by an automated colorimetric-enzymatic method on a Hitachi/Roche-Modular P analyzer (Basel, Switzerland). Insulin was measured by the immunometric ECLIA (Roche Modular E170). $\mathrm{HbA1c}$ was measured by Tosoh Automated Glycohemoglobin Analyzer HLC-723G8. Lipid levels were measured by the immunoassay analyzer Cobas 8000 (Roche, Basel, Switzerland). Coefficients of variance are $1 \%$ for glucose, $6 \%$ for insulin, about $2 \%$ for lipids and $2 \%$ for $\mathrm{HbA} 1 \mathrm{c}$ in the Lab of UZ Leuven.

\subsection{Statistical Analysis}

Descriptive statistics were given for the two groups where continuous variables are presented as means with standard deviation if normally distributed, or as medians with interquartile range otherwise; categorical variables are presented as frequencies with percentages. Logistic regression models were used for data analysis with postpartum glucose intolerance as a binary response variable 
and subject characteristics as explanatory variables. Results were reported as odds ratios (OR) with $95 \%$ confidence intervals. For variables during pregnancy that were significantly different between women with and without postpartum glucose intolerance, adjusted OR for age and BMI in early pregnancy were analyzed. The discriminative power of continuous variables for postpartum glucose intolerance is presented by receiver operating curve (ROC) curves and estimated as the area under the curve (AUC) with 95\% confidence interval. The AUC ranges between 0.5 (discrimination no better than chance) and 1 (perfect discrimination). Diagnostic accuracy of binary or dichotomized variables is estimated as sensitivity, specificity, positive and negative likelihood ratio, and post-test probability, with $95 \%$ confidence intervals. A $p$-value $<0.05$ (two-tailed) was considered significant. Analyses were performed by A. Laenen using SAS software (version 9.4).

\section{Results}

\subsection{Study Participants}

Prospective multicentric cohort study with 1841 women tested for GDM with a 75 g OGTT. GDM was diagnosed in $12.5 \%$ (231) of all participants. Of all women with GDM, 83.1\% (192) attended the postpartum $75 \mathrm{~g}$ OGTT. The OGTT was performed at $14.4 \pm 4.1$ weeks after delivery. Of all women with an OGTT postpartum, none had T2DM and 18.2\% (35) had glucose intolerance postpartum of which $37.1 \%$ (13) had IFG, 54.3\% (19) had IGT, and 8.6\% (3) had IFG and IGT combined.

\subsection{Characteristics of Women with Glucose Intolerance at the Time of the OGTT Postpartum}

Compared to NGT women, women with glucose intolerance postpartum were more insulin resistant [Matsuda index $0.50(0.33-0.83)$ vs. $0.74(0.48-1.08)$, OR $0.20(0.06-0.65), p=0.008$; HOMA-IR 16.8 (8.9-24.8) vs. 10.2 (7.3-15.6), OR $1.07(1.02-1.11), p=0.002]$, had a lower $\beta$-cell function [insulinogenic index/HOMA-IR $0.15(0.11-0.22)$ vs. $0.26(0.20-0.36)$, OR $0.001(0.00-0.07), p=0.001$ ], lower HDL-cholesterol [1.3 (1.1-1.5) vs. $1.5(1.3-1.8)$, OR $0.96(0.93-0.99), p=0.010]$, higher fasting triglycerides [1.2 (0.8-2.0) vs. $0.8(0.6-1.1) \mathrm{mmol} / \mathrm{L}$, OR $1.01(1.01-1.02), p<0.0001]$ and breastfed less often [66.7\% vs. $86.3 \%$, OR $0.32(0.13-0.75), p=0.009]$ (Table 1$)$. There was no difference in the duration of breastfeeding or in the rate of exclusive breastfeeding (Table 1). Women with glucose intolerance had a higher BMI compared to NGT women but this was borderline not significant $\left(28.1 \pm 6.6 \mathrm{~kg} / \mathrm{m}^{2}\right.$ vs. $\left.26.1 \pm 4.9 \mathrm{~kg} / \mathrm{m}^{2}, p=0.050\right)$.

Table 1. Characteristics of women with glucose intolerance compared to normal glucose tolerant women at the time of the OGTT postpartum.

\begin{tabular}{|c|c|c|c|c|}
\hline & $\begin{array}{l}\text { Glucose Intolerant } \\
\text { Postpartum } \\
18.2 \%(35)\end{array}$ & $\begin{array}{c}\text { NGT Postpartum } \\
\text { OGTT } \\
81.8 \%(157)\end{array}$ & OR $(95 \% \mathrm{CI})$ & $p$-Value \\
\hline $\mathrm{BMI}\left(\mathrm{kg} / \mathrm{m}^{2}\right)$ & $28.1 \pm 6.6$ & $26.1 \pm 4.9$ & $1.07(1.0-1.14)$ & 0.050 \\
\hline$\%$ overweight & $27.3(9)$ & $33.1(51)$ & $1.04(0.41-2.66)$ & 0.928 \\
\hline$\%$ Systolic hypertension & $6.1(2)$ & $4.5(7)$ & $1.35(0.27-6.84)$ & 0.713 \\
\hline Diastolic BP (mmHg) & $74.4 \pm 9.5$ & $73.2 \pm 9.0$ & $1.01(0.97-1.06)$ & 0.498 \\
\hline$\%$ Diastolic hypertension & $6.1(2)$ & $4.5(7)$ & $1.35(0.27-6.84)$ & 0.713 \\
\hline Waist circumference $(\mathrm{cm})$ & $92.7 \pm 12.5$ & $91.1 \pm 12.0$ & $1.01(0.98-1.04)$ & 0.50 \\
\hline
\end{tabular}


Table 1. Cont.

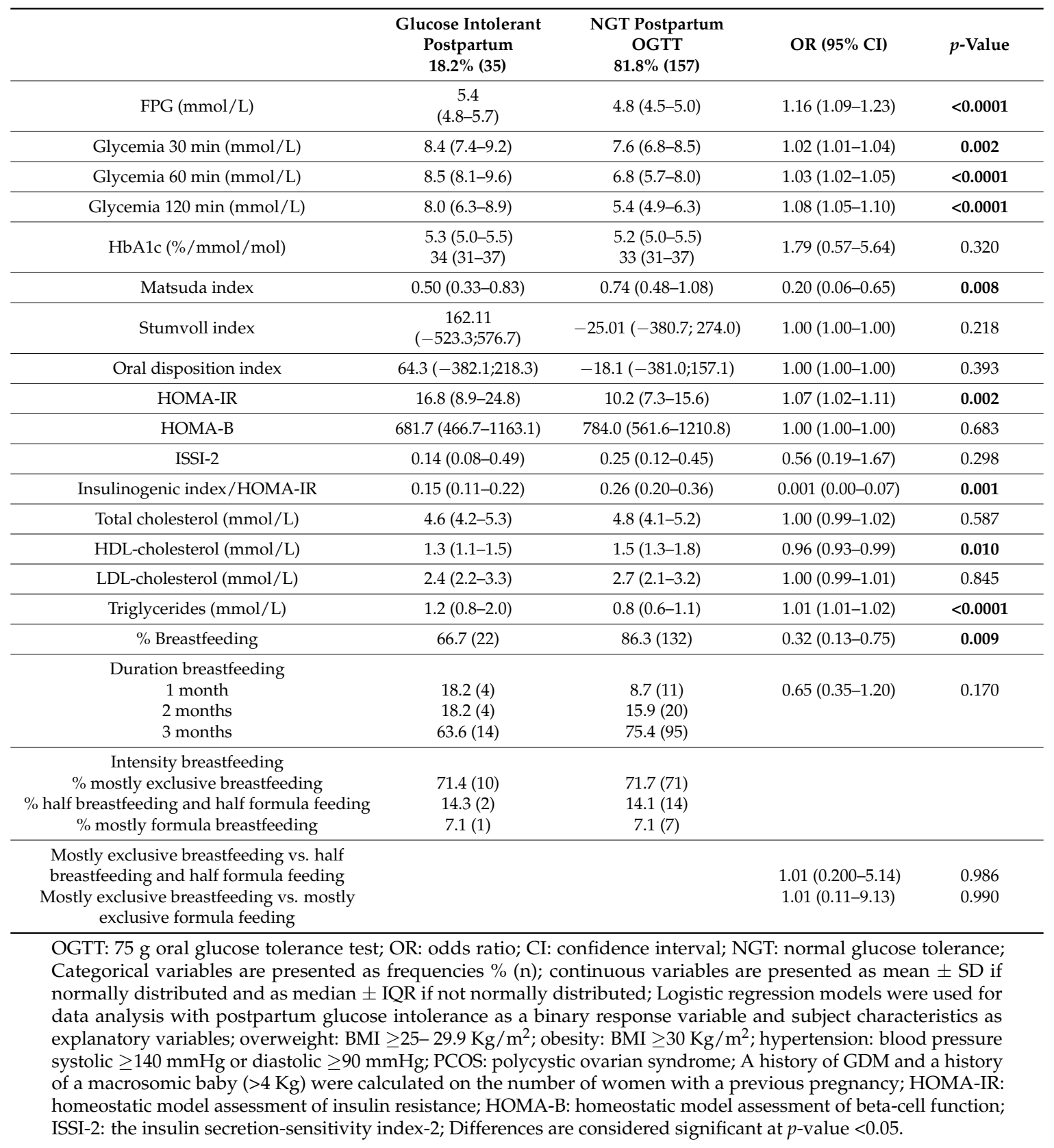

\subsection{Predictors for Glucose Intolerance Postpartum Based on Characteristics and Biochemical Variables Prepregnancy and during Pregnancy}

Based on the characteristics and biochemical variables in early and late pregnancy, compared to NGT women, women with glucose intolerance postpartum had more often an Asian origin $[15.1 \%$ vs. $3.7 \%$, OR $4.64(1.26-17.12), p=0.021$ ], were more often multiparous [ $68.6 \%$ vs. $48.4 \%$, OR 2.32 (1.07-5.07), $p=0.034]$, had more often a recurrent history of GDM [41.7\% vs. 26.7\%, OR 3.68 (1.37-9.87), $p=0.010]$, a higher fasting glycaemia (FPG) [5.1 (4.5-5.3) vs. $4.6(4.3-5.1) \mathrm{mmol} / \mathrm{L}, \mathrm{OR} 1.05(1.01-1.09)$, $p=0.023$ ], a higher HbA1c [33 (31-36) vs. 32 (30-33) $\mathrm{mmol} / \mathrm{mol}$, OR 4.89 (1.61-14.82), $p=0.005]$, and higher fasting triglycerides [2.2 (1.9-2.8) vs. $2.0(1.6-2.5) \mathrm{mmol} / \mathrm{L}, \mathrm{OR} 1.00(1.00-1.01), p=0.028]$ at the time of the OGTT during pregnancy (Tables 2-4). After adjustment for maternal age and BMI in early pregnancy, a recurrent history of GDM and FPG did not remain significant (Table 3). The AUC on the ROC curve for the $50 \mathrm{~g}$ GCT to predict glucose intolerance postpartum was 0.60 (CI 95\% 
0.449-0.71) (Figure 1). Evaluation of the sensitivity across different GCT thresholds, showed that a GCT $\geq 7.2 \mathrm{mmol} / \mathrm{L}$ during pregnancy had the highest sensitivity of $80 \%(95 \% \mathrm{CI} 63.1 \%-91.6 \%)$ with a specificity of $26.4 \%$ (95\% CI $14.6 \%-27.9 \%$ ) to predict glucose intolerance postpartum (Table 5).

Table 2. Predictors of glucose intolerance postpartum based on general characteristics and prepregnancy risk factors.

\begin{tabular}{|c|c|c|c|c|c|c|}
\hline & $\begin{array}{c}\text { Glucose } \\
\text { Intolerant } \\
\text { Postpartum } \\
\text { 18.2\% (35) }\end{array}$ & $\begin{array}{c}\text { NGT } \\
\text { Postpartum } \\
\text { OGTT 81.8\% } \\
(157)\end{array}$ & OR $(95 \%$ CI $)$ & $p$-Value & $\begin{array}{c}\text { Adjusted } \\
\text { OR }(95 \% \text { CI) }\end{array}$ & $p$-Value \\
\hline Age (years) & $32.7 \pm 4.4$ & $32.2 \pm 4.7$ & $\begin{array}{c}1.02 \\
(0.94-1.10)\end{array}$ & 0.595 & & \\
\hline$\%$ Non-Caucasian & $20.0(7)$ & $16.7(26)$ & $\begin{array}{c}1.25 \\
(0.49-3.16)\end{array}$ & 0.638 & & \\
\hline$\%$ Northern-African & $3.4(1)$ & $4.4(6)$ & $\begin{array}{c}0.77 \\
(0.09-6.68)\end{array}$ & 0.816 & & \\
\hline$\%$ Asian & $15.1(5)$ & $3.7(5)$ & $\begin{array}{c}4.64 \\
(1.26-17.12) \\
\end{array}$ & 0.021 & $\begin{array}{c}5.10 \\
(1.34-19.37)\end{array}$ & 0.017 \\
\hline Prepregnancy BMI $\left(\mathrm{kg} / \mathrm{m}^{2}\right)$ & $26.9 \pm 6.7$ & $25.6 \pm 5.4$ & $\begin{array}{c}1.04 \\
(0.97-1.11)\end{array}$ & 0.256 & & \\
\hline$\%$ overweight prepregnancy & $28.1(9)$ & $24.7(37)$ & $\begin{array}{c}1.56 \\
(0.60-4.03)\end{array}$ & 0.358 & & \\
\hline$\%$ obese prepregnancy & $28.1(9)$ & $20.7(31)$ & $\begin{array}{c}1.70 \\
(0.67-4.33)\end{array}$ & 0.265 & & \\
\hline$\%$ highest degree primary school & $2.9(1)$ & $1.4(2)$ & $\begin{array}{c}2.24 \\
(0.20-25.51) \\
\end{array}$ & 0.515 & & \\
\hline $\begin{array}{l}\% \text { highest degree lower secondary } \\
\text { school }\end{array}$ & $5.9(2)$ & $3.4(5)$ & $\begin{array}{c}1.79 \\
(0.33-9.68)\end{array}$ & 0.497 & & \\
\hline $\begin{array}{l}\% \text { no education higher than } \\
\text { secondary school }\end{array}$ & $18.2(6)$ & $23.2(33)$ & $\begin{array}{c}0.73 \\
(0.28-1.93) \\
\end{array}$ & 0.531 & & \\
\hline$\%$ no paid job & $11.8(4)$ & $7.1(11)$ & $\begin{array}{c}1.73 \\
(0.52-5.81)\end{array}$ & 0.373 & & \\
\hline$\%$ income from benefits & $2.9(1)$ & $1.9(3)$ & $\begin{array}{c}1.53 \\
(0.15-15.23)\end{array}$ & 0.714 & & \\
\hline
\end{tabular}

OR: odds ratio, adjusted OR (odds ratio) for maternal age and BMI in early pregnancy; CI: confidence interval; NGT: normal glucose tolerance; Categorical variables are presented as frequencies \% (n); continuous variables are presented as mean \pm SD if normally distributed and as median \pm IQR if not normally distributed; Logistic regression models were used for data analysis with postpartum glucose intolerance as a binary response variable and subject characteristics as explanatory variables; Differences are considered significant at $p$-value $<0.05$.

Table 3. Predictors of glucose intolerance postpartum based on clinical and biochemical risk factors 6-16 weeks of pregnancy.

\begin{tabular}{|c|c|c|c|c|c|c|}
\hline & $\begin{array}{c}\text { Glucose } \\
\text { Intolerant } \\
\text { Postpartum } \\
18.2 \%(35)\end{array}$ & $\begin{array}{c}\text { NGT } \\
\text { Postpartum } \\
\text { OGTT } 81.8 \% \\
(157)\end{array}$ & OR (95\% CI) & $p$-Value & $\begin{array}{c}\text { Adjusted } \\
\text { OR }(95 \% \text { CI) }\end{array}$ & $p$-Value \\
\hline $\mathrm{BMI}\left(\mathrm{kg} / \mathrm{m}^{2}\right)$ & $27.3 \pm 6.5$ & $26.4 \pm 5.1$ & $\begin{array}{c}1.03 \\
(0.96-1.10)\end{array}$ & 0.380 & & \\
\hline$\%$ overweight & $28.6(10)$ & $27.6(43)$ & $\begin{array}{c}1.18 \\
(0.49-2.85)\end{array}$ & 0.716 & & \\
\hline$\%$ obese & $28.6(10)$ & $23.1(36)$ & $\begin{array}{c}1.43 \\
(0.58-3.48)\end{array}$ & 0.436 & & \\
\hline Systolic BP (mmHg) & $114.9 \pm 13.9$ & $117.2 \pm 11.0$ & $0.98(0.95-1.0)$ & 0.284 & & \\
\hline \% Systolic hypertension & $8.6(3)$ & $1.9(3)$ & $\begin{array}{c}4.78 \\
(0.92-24.77)\end{array}$ & 0.062 & & \\
\hline Diastolic BP (mmHg) & $70.8 \pm 8.9$ & $72.5 \pm 8.9$ & $\begin{array}{c}0.98 \\
(0.94-1.02)\end{array}$ & 0.324 & & \\
\hline \% Diastolic hypertension & $5.7(2)$ & $3.2(5)$ & $\begin{array}{c}1.83 \\
(0.34-9.84)\end{array}$ & 0.481 & & \\
\hline Waist circumference $(\mathrm{cm})$ & $92.0 \pm 14.1$ & $90.3 \pm 12.8$ & $\begin{array}{c}1.01 \\
(0.98-1.04)\end{array}$ & 0.512 & & \\
\hline
\end{tabular}


Table 3. Cont.

\begin{tabular}{|c|c|c|c|c|c|c|}
\hline & $\begin{array}{c}\text { Glucose } \\
\text { Intolerant } \\
\text { Postpartum } \\
18.2 \%(35)\end{array}$ & $\begin{array}{c}\text { NGT } \\
\text { Postpartum } \\
\text { OGTT 81.8\% } \\
(157)\end{array}$ & OR $(95 \%$ CI $)$ & $p$-Value & $\begin{array}{c}\text { Adjusted } \\
\text { OR (95\% CI) }\end{array}$ & $p$-Value \\
\hline \multicolumn{7}{|l|}{$\%$ waist circumference } \\
\hline $80-88 \mathrm{~cm}$ & $34.4(11)$ & $31.8(48)$ & $\begin{array}{c}1.42 \\
(0.45-4.48)\end{array}$ & 0.549 & & \\
\hline$>88 \mathrm{~cm}$ & $50.0(16)$ & $47.7(72)$ & $\begin{array}{c}1.38 \\
(0.46-4.09)\end{array}$ & 0.564 & & \\
\hline$\%$ smoking before pregnancy & $8.6(3)$ & $3.2(5)$ & $\begin{array}{c}1.73 \\
(0.82-3.65) \\
\end{array}$ & 0.147 & & \\
\hline$\%$ smoking during pregnancy & $45.7(16)$ & $32.7(51)$ & $\begin{array}{c}2.83 \\
(0.64-12.45) \\
\end{array}$ & 0.168 & & \\
\hline$\%$ multiparity & $68.6(24)$ & $48.4(76)$ & $\begin{array}{c}2.32 \\
(1.07-5.07)\end{array}$ & 0.034 & $\begin{array}{c}2.37 \\
(1.06-5.31)\end{array}$ & 0.036 \\
\hline$\%$ history of miscarriage & $40.0(14)$ & $29.9(47)$ & $\begin{array}{c}1.56 \\
(0.73-3.33)\end{array}$ & 0.250 & & \\
\hline$\%$ history of GDM & $41.7(10 / 24)$ & $26.7(20 / 75)$ & $\begin{array}{c}3.68 \\
(1.37-9.87)\end{array}$ & 0.010 & $\begin{array}{c}1.89 \\
(0.72-4.97) \\
\end{array}$ & 0.197 \\
\hline$\%$ first degree family history of GDM & $6.2(2)$ & $6.2(9)$ & $\begin{array}{c}0.98 \\
(0.20-4.79) \\
\end{array}$ & 0.985 & & \\
\hline $\begin{array}{l}\% \text { first degree family history of } \\
\text { diabetes }\end{array}$ & $25.7(9)$ & $16.4(25)$ & $\begin{array}{c}1.71 \\
(0.68-4.25) \\
\end{array}$ & 0.251 & & \\
\hline$\%$ history of macrosomia & $12.5(3 / 24)$ & $15.8(13 / 76)$ & $\begin{array}{c}1.70 \\
(0.42-6.92) \\
\end{array}$ & 0.459 & & \\
\hline$\%$ history impaired glucose tolerance & $9.4(3)$ & $2.2(3)$ & $\begin{array}{c}0.22 \\
(0.04-1.13) \\
\end{array}$ & 0.069 & & \\
\hline$\%$ history of PCOS & $2.9(1)$ & $5.7(9)$ & $\begin{array}{c}2.07 \\
(0.25-16.87)\end{array}$ & 0.498 & & \\
\hline$\%$ fertility treatment & $25.7(9)$ & $17.2(27)$ & $\begin{array}{c}0.60 \\
(0.25-1.42) \\
\end{array}$ & 0.246 & & \\
\hline FPG (mmol/L) & $4.7(4.5-5.0)$ & $4.7(3.4-4.9)$ & $\begin{array}{c}0.10 \\
(0.95-1.05) \\
\end{array}$ & 0.928 & & \\
\hline$\% \mathrm{FPG} \geq 5.1 \mathrm{mmol} / \mathrm{L}$ & $11.8(4)$ & $13.5(21)$ & $\begin{array}{c}1.17 \\
(0.37-3.64) \\
\end{array}$ & 0.791 & & \\
\hline $\mathrm{HbA} 1 \mathrm{c}(\% / \mathrm{mmol} / \mathrm{mol})$ & $\begin{array}{l}5.1(4.9-5.3) \\
32(30-34)\end{array}$ & $\begin{array}{c}5.0(4.9-5.3) \\
31(30-34)\end{array}$ & $\begin{array}{c}0.734 \\
(0.23-2.33)\end{array}$ & 0.599 & & \\
\hline HOMA-IR & $10.2(7.2-17.6)$ & $10.7(7.6-16.9)$ & $\begin{array}{c}1.00 \\
(0.96-1.05)\end{array}$ & 0.839 & & \\
\hline HOMA-B & $\begin{array}{c}830.2 \\
(630.0-1284.0) \\
\end{array}$ & $\begin{array}{c}983.5 \\
(667.2-1393.6) \\
\end{array}$ & $\begin{array}{c}0.10 \\
(0.10-1.00) \\
\end{array}$ & 0.211 & & \\
\hline Total cholesterol (mmol/L) & $4.5(4.1-5.7)$ & $4.8(4.2-5.4)$ & $\begin{array}{c}1.00 \\
(0.99-1.01) \\
\end{array}$ & 0.805 & & \\
\hline HDL-cholesterol (mmol/L) & $1.7(1.3-1.9)$ & $1.7(1.5-2.0)$ & $\begin{array}{c}1.02 \\
(0.99-1.05)\end{array}$ & 0.197 & & \\
\hline LDL-cholesterol (mmol/L) & $2.2(1.9-3.1)$ & $2.4(2.1-2.9)$ & $\begin{array}{c}1.00 \\
(0.99-1.01)\end{array}$ & 0.887 & & \\
\hline Triglycerides (mmol/L) & $1.2(0.9-1.7)$ & $1.1(0.9-1.3)$ & $\begin{array}{c}0.99 \\
(0.99-1.00)\end{array}$ & 0.140 & & \\
\hline
\end{tabular}

OR: odds ratio, adjusted OR (odds ratio) for maternal age and BMI in early pregnancy; CI: confidence interval; NGT: normal glucose tolerance; Categorical variables are presented as frequencies $\%(n)$; continuous variables are presented as mean \pm SD if normally distributed and as median \pm IQR if not normally distributed; Logistic regression models were used for data analysis with postpartum glucose intolerance as a binary response variable and subject characteristics as explanatory variables; overweight: BMI $\geq 25-29.9 \mathrm{Kg} / \mathrm{m}^{2}$; obesity: BMI $\geq 30 \mathrm{Kg} / \mathrm{m}^{2}$; hypertension: blood pressure systolic $\geq 140 \mathrm{mmHg}$ or diastolic $\geq 90 \mathrm{mmHg}$; PCOS: polycystic ovarian syndrome; A history of GDM and a history of a macrosomic baby $(>4 \mathrm{Kg})$ were calculated on the number of women with a previous pregnancy; HOMA-IR: homeostatic model assessment of insulin resistance; HOMA-B: homeostatic model assessment of beta-cell function; ISSI-2: the insulin secretion-sensitivity index-2; Differences are considered significant at $p$-value $<0.05$. 
Table 4. Predictors of glucose intolerance postpartum based on clinical and biochemical risk factors 24-28 weeks of pregnancy.

\begin{tabular}{|c|c|c|c|c|c|c|}
\hline & $\begin{array}{c}\text { Glucose } \\
\text { Intolerant } \\
\text { Postpartum } \\
18.2 \%(35)\end{array}$ & $\begin{array}{c}\text { NGT } \\
\text { Postpartum } \\
\text { OGTT 81.8\% } \\
(157)\end{array}$ & OR $(95 \% \mathrm{CI})$ & $p$-Value & $\begin{array}{c}\text { Adjusted } \\
\text { OR }(95 \% \text { CI) }\end{array}$ & $p$-Value \\
\hline BMI $\left(\mathrm{kg} / \mathrm{m}^{2}\right)$ & $30.0 \pm 6.6$ & $28.9 \pm 4.8$ & $1.04(0.97-1.11)$ & 0.268 & & \\
\hline$\%$ overweight & $38.2(13)$ & $41.3(62)$ & $0.92(0.35-2.43)$ & 0.862 & & \\
\hline$\%$ obese & $38.2(13)$ & $35.3(53)$ & $1.07(0.40-2.85)$ & 0.888 & & \\
\hline Systolic BP (mmHg) & $115.3 \pm 11.8$ & $114.7 \pm 11.2$ & $1.00(0.97-1.04)$ & 0.767 & & \\
\hline$\%$ Systolic hypertension & $5.7(2)$ & $2.6(4)$ & $2.30(0.40-13.10)$ & 0.347 & & \\
\hline Diastolic BP $(\mathrm{mmHg})$ & $69.7 \pm 7.1$ & $68.7 \pm 8.4$ & $1.01(0.97-1.06)$ & 0.517 & & \\
\hline$\%$ Diastolic hypertension & $0.0(0)$ & $2.6(4)$ & 0.00 & 0.980 & & \\
\hline Fasting plasma glycemia (mmol/L) & $5.1(4.5-5.3)$ & $4.6(4.3-5.1)$ & $1.05(1.01-1.09)$ & 0.023 & $\begin{array}{c}1.04 \\
(1.00-1.09)\end{array}$ & 0.070 \\
\hline Glycemia $30 \mathrm{~min}$ (mmol/L) & $8.5(7.1-9.6)$ & $8.1(7.4-8.9)$ & $1.01(0.10-1.03)$ & 0.128 & & \\
\hline Glycemia $60 \mathrm{~min}(\mathrm{mmol} / \mathrm{L})$ & $9.7(8.3-10.5)$ & $9.6(8.7-10.3)$ & $1.00(0.98-1.01)$ & 0.974 & & \\
\hline Glycemia $120 \mathrm{~min}(\mathrm{mmol} / \mathrm{L})$ & $8.8(8.3-8.9)$ & $8.6(7.7-9.1)$ & $1.01(0.99-1.02)$ & 0.196 & & \\
\hline $\mathrm{HbA} 1 \mathrm{c}(\% / \mathrm{mmol} / \mathrm{mol})$ & $\begin{array}{l}5.2(5.0-5.4) \\
33(31-36)\end{array}$ & $\begin{array}{l}5.1(4.9-5.2) \\
32(30-33)\end{array}$ & 4.89 (1.61-14.82) & 0.005 & $\begin{array}{c}4.43 \\
(1.37-14.35)\end{array}$ & 0.013 \\
\hline$\%$ GCT $\geq 7.2 \mathrm{mmol} / \mathrm{L}$ & $80.00(28)$ & $73.5(114)$ & $1.44(0.58-3.54)$ & 0.430 & & \\
\hline Matsuda index & $0.30(0.21-0.50)$ & $0.40(0.25-0.52)$ & $0.40(0.06-2.69)$ & 0.349 & & \\
\hline Stumvoll index & $\begin{array}{c}529.3 \\
(32.5-1167.8)\end{array}$ & $\begin{array}{c}460.1 \\
(20.5-1154.4)\end{array}$ & $1.00(1.00-1.00)$ & 0.503 & & \\
\hline Oral disposition index & $\begin{array}{c}150.7 \\
(12.7-274.8)\end{array}$ & $\begin{array}{c}186.1 \\
(10.6-289.6)\end{array}$ & $1.00(0.10-1.00)$ & 0.421 & & \\
\hline HOMA-IR & $20.4(13.4-30.5)$ & $16.3(11.1-26.5)$ & $1.01(0.98-1.03)$ & 0.520 & & \\
\hline HOMA-B & $\begin{array}{c}1376.3 \\
(947.6-2047.1)\end{array}$ & $\begin{array}{c}1407.5 \\
(1055.1-2198.2)\end{array}$ & $1.00(1.00-1.00)$ & 0.429 & & \\
\hline ISSI-2 & $0.07(0.05-0.17)$ & $0.10(0.04-0.17)$ & $0.96(0.04-21.14)$ & 0.979 & & \\
\hline Insulinogenic index/HOMA-IR & $0.18(0.12-0.25)$ & $0.21(0.16-0.31)$ & $0.07(0.00-2.32)$ & 0.137 & & \\
\hline Total cholesterol (mg/dl) & $5.9(5.4-6.9)$ & $6.3(5.7-7.0)$ & $0.99(0.99-1.00)$ & 0.296 & & \\
\hline HDL-cholesterol (mmol/L) & $1.9(1.5-2.2)$ & $1.9(1.6-2.2)$ & $0.99(0.97-1.02)$ & 0.559 & & \\
\hline LDL-cholesterol (mmol/L) & $3.1(2.6-3.7)$ & $3.5(2.9-4.9)$ & $0.99(0.98-1.00)$ & 0.055 & & \\
\hline Triglycerides (mmol/L) & $2.2(1.9-2.8)$ & $2.0(1.6-2.5)$ & $1.00(1.00-1.01)$ & 0.028 & $\begin{array}{c}1.00 \\
(1.00-1.01)\end{array}$ & 0.049 \\
\hline Triglycerides change $(\mathrm{mmol} / \mathrm{L})$ & $0.9(0.6-1.5)$ & $0.9(0.6-1.2)$ & $1.01(1.00-1.01)$ & 0.082 & & \\
\hline Gestational weight gain $(\mathrm{Kg})$ & $9.1 \pm 5.1$ & $8.1 \pm 4.7$ & $1.04(0.96-1.13)$ & 0.299 & & \\
\hline$\%$ excessive gestational weight & $20.6(7)$ & $16.3(22)$ & $0.99(0.33-0.93)$ & 0.982 & & \\
\hline $\begin{array}{l}\text { Gestational age diagnosis GDM } \\
\text { (weeks) }\end{array}$ & $26.7 \pm 1.0$ & $26.9 \pm 1.1$ & $0.78(0.56-1.08)$ & 0.139 & & \\
\hline$\%$ insulin treatment & $25.7(9)$ & $13.4(21)$ & & 0.281 & & \\
\hline$\%$ Long-acting & $5.7(2)$ & $2.5(4)$ & $2.61(0.45-15.03)$ & 0.281 & & \\
\hline$\%$ short acting & $8.6(3)$ & $6.4(10)$ & $1.57(0.40-6.09)$ & 0.515 & & \\
\hline$\%$ short-and long acting & $11.4(4)$ & $4.7(7)$ & $2.10(0.82-10.95)$ & 0.098 & & \\
\hline \multicolumn{7}{|l|}{ Number of insulin injections } \\
\hline 1 & $22.2(2)$ & $33.3(7)$ & $1.29(0.65-2.56)$ & 0.459 & & \\
\hline 2 & $11.1(1)$ & $14.3(3)$ & & & & \\
\hline 3 & $33.3(3)$ & $28.6(6)$ & & & & \\
\hline 4 & $33.3(3)$ & $23.8(5)$ & & & & \\
\hline $\begin{array}{l}\text { Gestational age at start insulin } \\
\text { (weeks) }\end{array}$ & $28.6 \pm 2.6$ & $30.4 \pm 2.2$ & $0.67(0.43-1.04)$ & 0.074 & & \\
\hline Total dose insulin (units) & $22.0 \pm 15.4$ & $15.6 \pm 12.1$ & $1.05(0.98-1.12)$ & 0.132 & & \\
\hline
\end{tabular}

OR: odds ratio, adjusted OR (odds ratio) for maternal age and BMI in early pregnancy; CI: confidence interval; NGT: normal glucose tolerance; Categorical variables are presented as frequencies \% (n); continuous variables are presented as mean \pm SD if normally distributed and as median \pm IQR if not normally distributed; Logistic regression models were used for data analysis with postpartum glucose intolerance as a binary response variable and subject characteristics as explanatory variables; overweight: BMI $\geq 25-29.9 \mathrm{Kg} / \mathrm{m}^{2}$; obesity: BMI $\geq 30 \mathrm{Kg} / \mathrm{m}^{2}$; hypertension: blood pressure systolic $>140 \mathrm{mmHg}$ or diastolic $>90 \mathrm{mmHg}$; HOMA-IR: homeostatic model assessment of insulin resistance; HOMA-B: homeostatic model assessment of beta-cell function; ISSI-2: the insulin secretion-sensitivity index-2; Differences are considered significant at $p$-value $<0.05$. 


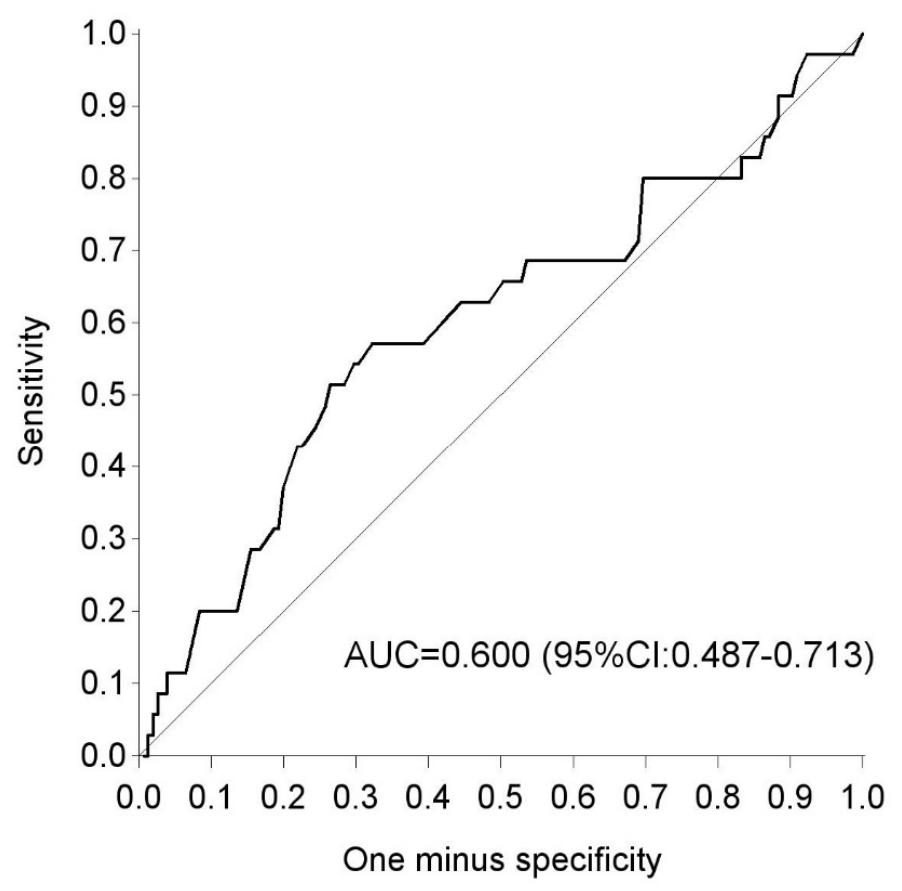

Figure 1. ROC curve for the glucose challenge test in pregnancy as a predictor for glucose intolerance postpartum. ROC: receiver operating curve; AUC: area under the curve.

Table 5. Sensitivity and specificity of the glucose challenge test for glucose intolerance postpartum.

\begin{tabular}{|c|c|c|c|c|c|c|}
\hline Threshold GCT & Sensitivity & Specificity & LR+ & LR- & $\begin{array}{c}\text { Positive } \\
\text { Post-test } \\
\text { Probability }\end{array}$ & $\begin{array}{c}\text { Negative } \\
\text { Post-test } \\
\text { Probability }\end{array}$ \\
\hline & $\begin{array}{c}\%(95 \% \mathrm{CI}) \\
\mathrm{n} / \mathrm{N}\end{array}$ & $\begin{array}{c}\%(95 \% \mathrm{CI}) \\
n / N\end{array}$ & (95\% CI) & $(95 \% \mathrm{CI})$ & $(95 \% \mathrm{CI})$ & $(95 \% \mathrm{CI})$ \\
\hline$\geq 7.8 \mathrm{mmol} / \mathrm{L}$ & $\begin{array}{c}68.6 \\
(50.7-83.1) \\
24 / 35\end{array}$ & $\begin{array}{c}41.3 \\
(33.4-49.5) \\
64 / 155\end{array}$ & $\begin{array}{c}1.2 \\
(0.9-1.5)\end{array}$ & $\begin{array}{c}0.76 \\
(0.45-1.29)\end{array}$ & $\begin{array}{c}20.6 \% \\
(12.9-29.4)\end{array}$ & $\begin{array}{c}14.5 \% \\
(8.9-21.5)\end{array}$ \\
\hline$\geq 7.5 \mathrm{mmol} / \mathrm{L}$ & $\begin{array}{c}68.6 \\
(50.7-83.1) \\
24 / 35\end{array}$ & $\begin{array}{c}32.9 \\
(25.6-40.9) \\
51 / 155\end{array}$ & $\begin{array}{c}1.0 \\
(0.8-1.3)\end{array}$ & $\begin{array}{c}0.96 \\
(0.56-1.64)\end{array}$ & $\begin{array}{c}18.5 \% \\
(11.7-26.6)\end{array}$ & $\begin{array}{c}17.5 \% \\
(10.8-25.5)\end{array}$ \\
\hline$\geq 7.2 \mathrm{mmol} / \mathrm{L}$ & $\begin{array}{c}80.0 \\
(63.1-91.6) \\
28 / 35\end{array}$ & $\begin{array}{c}26.4 \\
(19.7-34.1) \\
41 / 155\end{array}$ & $\begin{array}{c}1.1 \\
(0.9-1.3)\end{array}$ & $\begin{array}{c}0.76 \\
(0.37-1.54)\end{array}$ & $\begin{array}{c}19.5 \% \\
(12.3-27.9)\end{array}$ & $\begin{array}{c}14.4 \% \\
(8.4-21.7)\end{array}$ \\
\hline$\geq 6.9 \mathrm{mmol} / \mathrm{L}$ & $\begin{array}{c}80.0 \\
(63.1-91.6) \\
28 / 35\end{array}$ & $\begin{array}{c}20.6 \\
(14.6-27.9) \\
32 / 155\end{array}$ & $\begin{array}{c}1.0 \\
(0.8-1.2)\end{array}$ & $\begin{array}{c}0.97 \\
(0.47-2.01)\end{array}$ & $\begin{array}{c}18.3 \% \\
(11.6-26.3)\end{array}$ & $\begin{array}{c}17.7 \% \\
(10.6-26.1)\end{array}$ \\
\hline$\geq 6.7 \mathrm{mmol} / \mathrm{L}$ & $\begin{array}{c}80.0 \\
(63.1-91.6) \\
28 / 35\end{array}$ & $\begin{array}{c}16.8 \\
(11.3-23.6) \\
26 / 155\end{array}$ & $\begin{array}{c}1.0 \\
(0.8-1.1)\end{array}$ & $\begin{array}{c}1.19 \\
(0.56-2.52)\end{array}$ & $\begin{array}{c}17.6 \% \\
(11.2-25.3)\end{array}$ & $\begin{array}{c}21.0 \% \\
(12.6-30.4)\end{array}$ \\
\hline
\end{tabular}

GCT: 50 g glucose challenge test; CI: confidence interval; Sensitivity: $n=$ number with GCT $\geq$ cut-off; $N=$ number with glucose intolerance postpartum; Specificity: $\mathrm{n}=$ number with GCT < cut-off; $\mathrm{N}=$ number without glucose intolerance postpartum; LR+: positive likelihood ratio; LR-: negative likelihood ratio.

\subsection{Fasting Glycaemia and Hba1c Postpartum to Detect Glucose in Tolerance}

The AUC for FPG alone in early postpartum to predict glucose intolerance was 0.76 (95\% CI 0.65-0.87), the AUC for HbA1c alone was 0.54 (95\% CI 0.43-0.65) and the AUC for FPG and Hba1c combined was 0.75 (95\% CI 0.64-0.86) (Figure 2). 


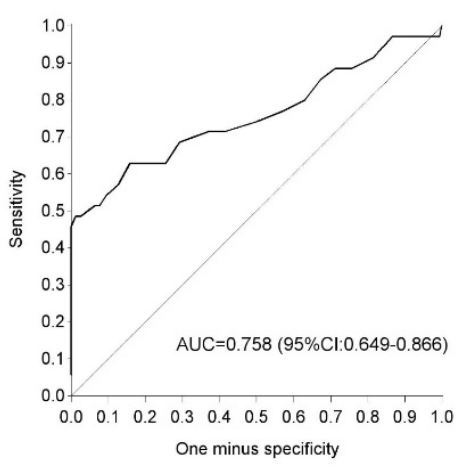

(A)

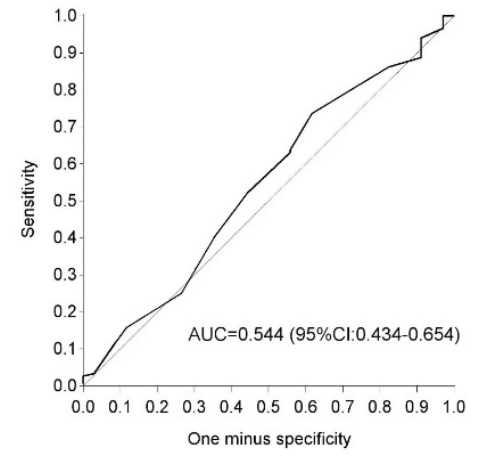

(B)

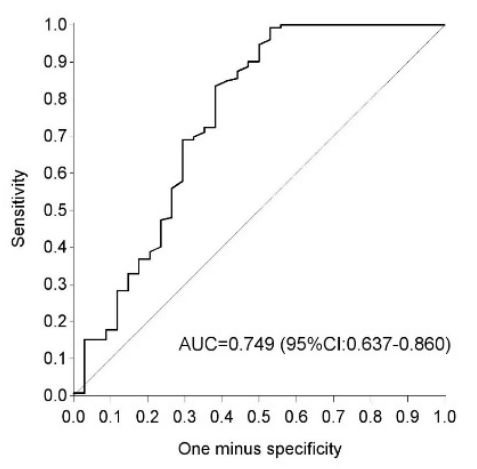

(C)

Figure 2. ROC curve for fasting plasma glucose and $\mathrm{HbA} 1 \mathrm{c}$ postpartum as a predictor for glucose intolerance. ROC: receiver operating curve; AUC: area under the curve; (A) ROC curve for fasting plasma glucose alone; (B) ROC curve for HbA1c alone; (C) ROC curve for fasting plasma glucose and $\mathrm{HbA1c}$ combined.

\section{Discussion}

By diagnosing GDM, a group of women at high risk to develop T2DM and cardiovascular disease later in life was identified [4,5]. Lifestyle interventions and metformin can prevent progression to T2DM on the long-term [24]. However, in normal routine attendance rates at screening tests postpartum are often low, with only $30 \%-50 \%$ of women with recent GDM receiving an OGTT or even a FPG within one year after the delivery and follow-up rates after one year dropping further $[25,26]$. Even in prospective studies, it remains challenging to obtain high attendance rates as demonstrated in the present study, where nearly one fifth of women with GDM did not attend the OGTT in early postpartum. This is a missed opportunity to timely identify and treat high-risk women for glucose intolerance. A GDM recall register implemented in the northern part of Belgium (Flanders), helps to successfully stimulate screening postpartum in primary care by sending annual reminders to women with a history of GDM [7]. This is in contrast with a South Australian GDM recall register were the response was limited [27]. However, in the South Australian GDM recall register, women who did not respond to a reminder letter were not followed up for a response while the Flemish register did not only use annual reminders by letters and email but if needed, additional reminders by telephone or text messages were used to increase the response rate [7]. Compliance with postpartum screening might also improve if an FPG and/or HbA1c could be used to screen for glucose intolerance instead of the cumbersome OGTT. However, several studies have shown that an FPG or Hba1c alone in early postpartum would miss the majority of women with glucose intolerance $[11,28,29]$. This is in line with our results showing that the majority of women with glucose intolerance had IGT. IGT is an important risk factor for T2DM, since the highest cumulative incidence to develop T2DM is seen in people with combined IFG/IGT, followed by isolated IGT with the lowest incidence in patients with 
IFG [30]. Moreover, we show now that combining an FPG and HbA1c in early postpartum, does not improve the accuracy to detect glucose intolerance compared to an FPG alone.

Data on the risk to develop T2DM in women with GDM diagnosed by the one-step approach and 2013 WHO criteria are limited. Before the introduction of the 2013 WHO screening strategy, studies have shown that $30 \%-50 \%$ of women with GDM develop T2DM within the first 10 years after the index pregnancy [4]. The use of the $2013 \mathrm{WHO}$ criteria for GDM results in a greater proportion of women who are diagnosed with mild forms of GDM, which might lead to a lower proportion at risk for postpartum glucose intolerance compared to women diagnosed with GDM by a two-step screening strategy. We show that nearly one-fifth of women with GDM based on a universal one-step screening strategy with the 2013 WHO criteria have glucose intolerance in early postpartum. An Irish study showed a rate of $\mathrm{T} 2 \mathrm{DM}$ of $2.2 \%$ and prediabetes of $23.7 \%$ up to 5 years post-delivery in women with GDM based on the $2013 \mathrm{WHO}$ criteria [12,31]. In contrast, we have previously shown glucose intolerance in $42 \%$ three months postpartum in women with GDM diagnosed by a two-step screening strategy with the 2013 WHO criteria [11]. Follow-up of the HAPO study 10-14 years postpartum, showed that untreated women with GDM, defined post hoc by the 2013 WHO criteria, had significantly higher rates of a disorder of glucose metabolism than women without GDM [52.2\% vs. 20.1\% (T2DM $10.7 \%$ vs. $1.6 \%)$ [ [32]. However, GDM according to the Carpenter \& Coustan criteria as defined in the HAPO follow-up study, was associated with a much higher risk for T2DM ( $20 \%$ vs. $7.9 \%)$ compared to women with GDM according to the 2013 WHO criteria alone [32]. The high prevalence of glucose intolerance postpartum using the Carpenter \& Coustan criteria for GDM is to be expected since these criteria were developed to identify women at high risk for the development of T2DM after the delivery [33].

Finding the risk factors for glucose intolerance postpartum after a recent history of GDM is important to identify a subgroup of women at a particularly high risk to progress to T2DM on the long-term. A more intensive follow-up and treatment of this high risk group might be cost-effective to prevent T2DM [34]. The most important risk factors to develop glucose intolerance in early postpartum differ according to the populations studied [35]. A systematic review has shown that BMI, family history of diabetes, non-white ethnicity, advanced maternal age, early diagnosis of GDM, raised FPG and $\mathrm{HbA1c}$, and insulin use during pregnancy are associated with future risk of T2DM [36]. This is in contrast to our study, showing that only an Asian origin, multiparity, a recurrent history of GDM and increased FPG, $\mathrm{HbA} 1 \mathrm{c}$ and fasting triglycerides in pregnancy, are risk factors for glucose intolerance in early postpartum. These predictors could be used to identify a subgroup of women with GDM with the highest risk to develop glucose intolerance postpartum and as such help to individualize the intensity of the follow-up. However, other common risk factors such as maternal age and BMI in pregnancy did not emerge as predictors in our study. This might be due to high maternal age of our GDM cohort and the fact that about $50 \%$ of GDM women were overweight or obese. Moreover, differences in screening strategy and diagnostic criteria used for GDM between different studies make comparisons difficult. In addition, since women were screened for GDM between 24-28 weeks of pregnancy, we could not evaluate whether testing for GDM before 24 weeks of pregnancy, might identify a group at higher risk for glucose intolerance postpartum.

As can be expected, we show that women with GDM who developed glucose intolerance in early postpartum were more insulin resistant and had an impaired beta-cell function compared to NGT women after delivery. During pregnancy insulin sensitivity and beta-cell dysfunction were not significantly different between both groups. However, women who develop GDM, often already have a subclinical metabolic dysfunction prior to conception compared with NGT women [37]. We speculate that the subgroup of GDM women at the highest risk for T2DM on the long-term, probably already have important predisposing baseline insulin resistance and/or beta-cell dysfunction present before pregnancy.

In our study, women with glucose intolerance postpartum breastfed less often than NGT women but without a difference in the duration of breastfeeding or in the exclusivity of breastfeeding between both groups. However, the timing of the postpartum OGTT varied between participants and we have 
no prospective data to evaluate the association between the duration of breastfeeding and risk for glucose intolerance postpartum. In addition, we have no data on breastfeeding beyond three months after delivery. There is now strong evidence that lactation duration ( $>3-6$ months) is independently associated with a graded reduction in the incidence of T2DM [12,38]. This might be due to the lower fasting and postprandial glucose levels and lower insulin secretion seen in lactating women [38].

Finally, we show that a GCT can also have a role in predicting postpartum glucose intolerance. We have previously shown that a GCT threshold of $7.2 \mathrm{mmol} / \mathrm{L}$ has the best trade-off between sensitivity and specificity to screen for GDM when using the 2013 WHO criteria [14]. Here we show that a GCT threshold of $7.2 \mathrm{mmol} / \mathrm{L}$ has also the best sensitivity to predict glucose intolerance in early postpartum. A two-step screening strategy with a GCT has therefore the potential to limit the number of OGTTs to screen for GDM based on the 2013 WHO criteria and at the same time identify a high risk group for glucose intolerance postpartum.

The strengths of our study are the large prospective multiethnic cohort with the availability of many clinical and biochemical variables in early pregnancy. In addition, we provide data on the predictive value of $\mathrm{HbA} 1 \mathrm{c}$ and FPG in early postpartum to detect glucose intolerance. Moreover, we present novel data on the predictive value of a GCT when used in a two-step screening strategy for GDM with the 2013 WHO criteria to predict glucose intolerance postpartum. The limitations are the lack of a control group postpartum and the lack of long-term data postpartum.

In conclusion, we show that nearly one-fifth of women with GDM based on the 2013 WHO criteria have glucose intolerance in early postpartum and these women have a more adverse metabolic profile. An FPG and HbA1c in early postpartum are not accurate enough to replace a $75 \mathrm{~g}$ OGTT to detect glucose intolerance. In addition, a GCT threshold of $7.2 \mathrm{mmol} / \mathrm{L}$ has the best sensitivity to predict glucose intolerance in early postpartum. Combining CGT and risk factors identified in the present study could identify a group of women who are at the highest risk for postpartum glucose intolerance and where recall incentives may be most needed.

Author Contributions: Conceptualization, K.B., P.V.C., and C.M. (Chantal Mathieu); Data curation, C.M. (Carolien Moyson); Formal analysis, A.L. (Annouschka Laenen); Funding acquisition, K.B., P.V.C., and C.M. (Chantal Mathieu); Investigation, K.B., P.V.C., J.V., S.V., H.V., C.V., T.M., E.D., C.D.B., Y.J., F.M., K.D.C., A.V.D.B., A.L. (Anne Loccufier), R.D., and C.M. (Chantal Mathieu); Methodology, K.B., P.V.C., and C.M. (Chantal Mathieu); Project administration, K.B. and C.M. (Carolien Moyson); Resources, K.B.; Supervision, K.B. and C.M. (Chantal Mathieu); Visualization, C.M. (Caro Minschart); Writing—original draft, K.B.; Writing—review \& editing, K.B., P.V.C., C.M. (Carolien Moyson), J.V., S.V., H.V., C.V., T.M., E.D., C.D.B., Y.J., F.M., K.D.C., A.V.D.B., A.L. (Anne Loccufier), A.L. (Annouschka Laenen), C.M. (Caro Minschart), R.D., and C.M. (Chantal Mathieu).

Funding: This investigator-initiated study was funded by the Belgian National Lottery, the Fund of Academic studies of UZ Leuven and the Fund Yvonne and Jacques François-de Meurs of the King Boudewijn Foundation.

Acknowledgments: K.B. is the recipient of a Clinical Doctoral Scholarship of the academic fund of UZ Leuven, and R.D. is the recipient of a 'Fundamenteel Klinisch Navorserschap FWO Vlaanderen'. We thank Inge Beckstedde from the UZA site and Sylva Van Imschoot from the AZ St Jan Brugge site for their help with the recruitment and study assessments. We thank the research assistants, paramedics, and physicians of all participating centers for their support and we thank all women who participated in the study.

Conflicts of Interest: The authors declare no conflict of interest. The funders of the study had no role in the design of the study; or in the collection, handling, analysis; or interpretation of the data; or in the decision to write and submit the manuscript for publication.

\section{References}

1. American Diabetes Association. Standards of medical care in diabetes-2013. Diabetes Care 2013, 36 (Suppl. 1), S11-S66. [CrossRef]

2. Crowther, C.A.; Hiller, J.E.; Moss, J.R.; McPhee, A.J.; Jeffries, W.S.; Robinson, J.S. Effect of treatment of gestational diabetes mellitus on pregnancy outcomes. N. Engl. J. Med. 2005, 352, 2477-2486. [CrossRef]

3. Landon, M.B.; Spong, C.Y.; Thom, E.; Carpenter, M.W.; Ramin, S.M.; Casey, B.; Wapner, R.J.; Varner, M.W.; Rouse, D.J.; Thorp, J.M., Jr.; et al. A multicenter, randomized trial of treatment for mild gestational diabetes. N. Engl. J. Med. 2009, 361, 1339-1348. [CrossRef] 
4. Bellamy, L.; Casas, J.P.; Hingorani, A.D.; Williams, D. Type 2 diabetes mellitus after gestational diabetes: A systematic review and meta-analysis. Lancet 2009, 373, 1773-1779. [CrossRef]

5. Song, C.; Lyu, Y.; Li, C.; Liu, P.; Li, J.; Ma, R.C.; Yang, X. Long-term risk of diabetes in women at varying durations after gestational diabetes: A systematic review and meta-analysis with more than 2 million women. Obes. Rev. 2018, 19, 421-429. [CrossRef]

6. Gerstein, H.C.; Santaguida, P.; Raina, P.; Morrison, K.M.; Balion, C.; Hunt, D.; Yazdi, H.; Booker, L. Annual incidence and relative risk of diabetes in people with various categories of dysglycemia: A systematic overview and meta-analysis of prospective studies. Diabetes Res. Clin. Pract. 2007, 78, 305-312. [CrossRef] [PubMed]

7. Benhalima, K.; Verstraete, S.; Muylle, F.; Decochez, K.; Devlieger, R.; Crombrugge, P.V.; Verhaegen, A.; Wens, J.; Mathieu, C. Implementing a Reminder System in the Northern Part of Belgium to Stimulate Postpartum Screening for Glucose Intolerance in Women with Gestational Diabetes: The "Sweet Pregnancy" Project. Int. J. Endocrinol. 2017, 2017, 3971914. [CrossRef]

8. International Association of Diabetes and Pregnancy Study Groups Consensus Panel; Metzger, B.E.; Gabbe, S.G.; Persson, B.; Buchanan, T.A.; Catalano, P.A.; Damm, P.; Dyer, A.R.; Leiva, A. International association of diabetes and pregnancy study groups recommendations on the diagnosis and classification of hyperglycemia in pregnancy. Diabetes Care 2010, 33, 676-682.

9. Diagnostic criteria and classification of hyperglycaemia first detected in pregnancy: A World Health Organization Guideline. Diabetes Res. Clin. Pract. 2014, 103, 341-363. [CrossRef]

10. Group, H.S.C.R.; Metzger, B.E.; Lowe, L.P.; Dyer, A.R.; Trimble, E.R.; Chaovarindr, U.; Coustan, D.R.; Hadden, D.R.; McCance, D.R.; Hod, M.; et al. Hyperglycemia and adverse pregnancy outcomes. N. Engl. J. Med. 2008, 358, 1991-2002.

11. Benhalima, K.; Jegers, K.; Devlieger, R.; Verhaeghe, J.; Mathieu, C. Glucose Intolerance after a Recent History of Gestational Diabetes Based on the 2013 WHO Criteria. PLoS ONE 2016, 11, e0157272. [CrossRef] [PubMed]

12. O'Reilly, M.W.; Avalos, G.; Dennedy, M.C.; O'Sullivan, E.P.; Dunne, F. Atlantic DIP: High prevalence of abnormal glucose tolerance post partum is reduced by breast-feeding in women with prior gestational diabetes mellitus. Eur. J. Endocrinol./Eur. Fed. Endocr. Soc. 2011, 165, 953-959.

13. Benhalima, K.; Van Crombrugge, P.; Verhaeghe, J.; Vandeginste, S.; Verlaenen, H.; Vercammen, C.; Dufraimont, E.; De Block, C.; Jacquemyn, Y.; Mekahli, F.; et al. The Belgian Diabetes in Pregnancy Study (BEDIP-N), a multi-centric prospective cohort study on screening for diabetes in pregnancy and gestational diabetes: Methodology and design. BMC Pregnancy Childbirth 2014, 14, 226. [CrossRef]

14. Benhalima, K.; Van Crombrugge, P.; Moyson, C.; Verhaeghe, J.; Vandeginste, S.; Verlaenen, H.; Vercammen, C.; Maes, T.; Dufraimont, E.; De Block, C.; et al. The Sensitivity and Specificity of the Glucose Challenge Test in a Universal Two-Step Screening Strategy for Gestational Diabetes Mellitus Using the 2013 World Health Organization Criteria. Diabetes Care 2018, 41, e111-e112. [CrossRef] [PubMed]

15. Benhalima, K.; Van Crombrugge, P.; Moyson, C.; Verhaeghe, J.; Vandeginste, S.; Verlaenen, H.; Vercammen, C.; Maes, T.; Dufraimont, E.; De Block, C.; et al. A Modified Two-Step Screening Strategy for Gestational Diabetes Mellitus Based on the 2013 WHO Criteria by Combining the Glucose Challenge Test and Clinical Risk Factors. J. Clin. Med. 2018, 7, 351. [CrossRef]

16. National Institute of Medicine Guidelines. Weight Gain During Pregnancy: Reexamining the Guidelines. In The National Academies Collection: Reports Funded by National Institutes of Health; Rasmussen, K.M., Ed.; National Academies Press (US): Washington, DC, USA, 2009.

17. Matthews, D.R.; Hosker, J.P.; Rudenski, A.S.; Naylor, B.A.; Treacher, D.F.; Turner, R.C. Homeostasis model assessment: Insulin resistance and $\beta$-cell function from fasting plasma glucose and insulin concentrations in man. Diabetologia 1985, 28, 412-419. [CrossRef]

18. Matsuda, M.; DeFronzo, R.A. Insulin sensitivity indices obtained from oral glucose tolerance testing: Comparison with the euglycemic insulin clamp. Diabetes Care 1999, 22, 1462-1470. [CrossRef] [PubMed]

19. Kahn, S.E. The relative contributions of insulin resistance and $\beta$-cell dysfunction to the pathophysiology of Type 2 diabetes. Diabetologia 2003, 46, 3-19. [CrossRef]

20. Kirwan, J.P.; Huston-Presley, L.; Kalhan, S.C.; Catalano, P.M. Clinically useful estimates of insulin sensitivity during pregnancy: Validation studies in women with normal glucose tolerance and gestational diabetes mellitus. Diabetes Care 2001, 24, 1602-1607. [CrossRef] [PubMed] 
21. Retnakaran, R.; Qi, Y.; Goran, M.I.; Hamilton, J.K. Evaluation of proposed oral disposition index measures in relation to the actual disposition index. Diabet. Med. 2009, 26, 1198-1203. [CrossRef] [PubMed]

22. Stumvoll, M.; Van Haeften, T.; Fritsche, A.; Gerich, J. Oral glucose tolerance test indexes for insulin sensitivity and secretion based on various availabilities of sampling times. Diabetes Care 2001, 24, 796-797. [CrossRef] [PubMed]

23. Elbein, S.C.; Wegner, K.; Kahn, S.E. Reduced $\beta$-cell compensation to the insulin resistance associated with obesity in members of caucasian familial type 2 diabetic kindreds. Diabetes Care 2000, 23, 221-227. [CrossRef]

24. Aroda, V.R.; Christophi, C.A.; Edelstein, S.L.; Zhang, P.; Herman, W.H.; Barrett-Connor, E.; Delahanty, L.M.; Montez, M.G.; Ackermann, R.T.; Zhuo, X.; et al. The effect of lifestyle intervention and metformin on preventing or delaying diabetes among women with and without gestational diabetes: The Diabetes Prevention Program outcomes study 10-year follow-up. J. Clin. Endocrinol. Metab. 2015, 100, 1646-1653. [CrossRef]

25. Ferrara, A.; Peng, T.; Kim, C. Trends in postpartum diabetes screening and subsequent diabetes and impaired fasting glucose among women with histories of gestational diabetes mellitus: A report from the Translating Research Into Action for Diabetes (TRIAD) Study. Diabetes Care 2009, 32, 269-274. [CrossRef] [PubMed]

26. Lawrence, J.M.; Black, M.H.; Hsu, J.W.; Chen, W.; Sacks, D.A. Prevalence and timing of postpartum glucose testing and sustained glucose dysregulation after gestational diabetes mellitus. Diabetes Care 2010, 33, 569-576. [CrossRef] [PubMed]

27. Chittleborough, C.R.; Baldock, K.L.; Taylor, A.W.; Hague, W.M.; Willson, T.; Martin, W.; Wood, J.; Phillips, P.J. Long-term follow-up of women with gestational diabetes mellitus: The South Australian Gestational Diabetes Mellitus Recall Register. Aust. New Zealand J. Obstet. Gynaecol. 2010, 50, 127-131. [CrossRef] [PubMed]

28. Kapaya, H.; May, L.; Jacques, R.; Madhuvrata, P. Sensitivity of postnatal fasting plasma glucose in identifying impaired glucose tolerance in women with gestational diabetes-25 Years' data. Obstet. Med. 2017, 10, 125-131. [CrossRef] [PubMed]

29. Kim, K.S.; Kim, S.K.; Cho, Y.W.; Park, S.W. Diagnostic value of haemoglobin A1c in post-partum screening of women with gestational diabetes mellitus. Diabet. Med. J. Br. Diabet. Assoc. 2016, 33, 1668-1672. [CrossRef]

30. Richter, B.; Hemmingsen, B.; Metzendorf, M.I.; Takwoingi, Y. Development of type 2 diabetes mellitus in people with intermediate hyperglycaemia. Cochrane Database Syst. Rev. 2018, 10, CD012661. [CrossRef]

31. Noctor, E.; Crowe, C.; Carmody, L.A.; Avalos, G.M.; Kirwan, B.; Infanti, J.J.; O’Dea, A.; Gillespie, P.; Newell, J.; McGuire, B.; et al. ATLANTIC DIP: Simplifying the follow-up of women with previous gestational diabetes. Eur. J. Endocrinol./Eur. Fed. Endocr. Soc. 2013, 169, 681-687. [CrossRef] [PubMed]

32. Lowe, W.L., Jr.; Scholtens, D.M.; Lowe, L.P.; Kuang, A.; Nodzenski, M.; Talbot, O.; Catalano, P.M.; Linder, B.; Brickman, W.J.; Clayton, P.; et al. Association of Gestational Diabetes With Maternal Disorders of Glucose Metabolism and Childhood Adiposity. JAMA 2018, 320, 1005-1016. [CrossRef] [PubMed]

33. Metzger, B.E.; Buchanan, T.A.; Coustan, D.R.; de Leiva, A.; Dunger, D.B.; Hadden, D.R.; Hod, M.; Kitzmiller, J.L.; Kjos, S.L.; Oats, J.N.; et al. Summary and recommendations of the Fifth International Workshop-Conference on Gestational Diabetes Mellitus. Diabetes Care 2007, 30 (Suppl. 2), S251-S260. [CrossRef] [PubMed]

34. Werbrouck, A.; Schmidt, M.; Putman, K.; Benhalima, K.; Verhaeghe, N.; Annemans, L.; Simoens, S. A systematic review on costs and cost-effectiveness of screening and prevention of type 2 diabetes in women with prior gestational diabetes: Exploring uncharted territory. Diabetes Res. Clin. Pract. 2018, 147, 138-148. [CrossRef] [PubMed]

35. Leuridan, L.; Wens, J.; Devlieger, R.; Verhaeghe, J.; Mathieu, C.; Benhalima, K. Glucose intolerance in early postpartum in women with gestational diabetes: Who is at increased risk? Prim. Care Diabetes 2015, 9, 244-252. [CrossRef]

36. Rayanagoudar, G.; Hashi, A.A.; Zamora, J.; Khan, K.S.; Hitman, G.A.; Thangaratinam, S. Quantification of the type 2 diabetes risk in women with gestational diabetes: A systematic review and meta-analysis of 95,750 women. Diabetologia 2016, 59, 1403-1411. [CrossRef] [PubMed] 
37. Catalano, P.M. Trying to understand gestational diabetes. Diabet. Med. J. Br. Diabet. Assoc. 2014, 31, $273-281$. [CrossRef] [PubMed]

38. Gunderson, E.P.; Lewis, C.E.; Lin, Y.; Sorel, M.; Gross, M.; Sidney, S.; Jacobs, D.R., Jr.; Shikany, J.M.; Quesenberry, C.P., Jr. Lactation Duration and Progression to Diabetes in Women Across the Childbearing Years: The 30-Year CARDIA Study. JAMA Intern. Med. 2018, 178, 328-337. [CrossRef] [PubMed]

(C) 2019 by the authors. Licensee MDPI, Basel, Switzerland. This article is an open access article distributed under the terms and conditions of the Creative Commons Attribution (CC BY) license (http:/ / creativecommons.org/licenses/by/4.0/). 